\title{
ON STABLE UNIQUENESS IN LINEAR SEMI-INFINITE OPTIMIZATION $^{1}$
}

\author{
M.A. Goberna ${ }^{2}$, M.I. Todorov ${ }^{3}$, and V.N. Vera de Serio ${ }^{4}$
}

\begin{abstract}
This paper is intended to provide conditions for the stability of the strong uniqueness of the optimal solution of a given linear semi-infinite optimization (LSIO) problem, in the sense of the maintaining of the strong uniqueness property under sufficiently small perturbations of all the data. We consider LSIO problems such that the family of gradients of all the constraints is unbounded, extending earlier results of Nürnberger for continuous LSIO problems, and of Helbig and Todorov for LSIO problems with bounded set of gradients. To do this we characterize the absolutely (affinely) stable problems, i.e., those LSIO problems whose feasible set (its affine hull, respectively) remains constant under sufficiently small perturbations.

Key words. Linear semi-infinite optimization, stable strong uniqueness, extended Nürnberger condition, affine stability, absolute stability.
\end{abstract}

2000 Mathematics Subject Classification. 90C05, 90C34, 15 A39.

\section{Introduction}

This paper deals with linear semi-infinite optimization (LSIO) problems in $\mathbb{R}^{n}$ of the form

$$
\pi: \text { Inf } c^{\prime} x \text { s.t. } a_{t}^{\prime} x \geq b_{t}, t \in T,
$$

where $T$ is a fixed arbitrary (possible infinite) set, $a: T \rightarrow \mathbb{R}^{n}$ and $b: T \rightarrow \mathbb{R}$ are functions, and $x \in \mathbb{R}^{n}$. It is focussed on the identification of those LSIO problems which have a strongly unique (or just unique) optimal solution and this desirable property is stable in the sense that it is preserved by sufficiently small perturbations of the cost vector $c$ and the functions $a$ and $b$. For short,

$\overline{1}$ This work has been supported by MICINN of Spain, Grant MTM2008-06695C03-01/03, by Generalitat Valenciana, by CONACyT of MX, Grant 55681, and by SECTyP-UNCuyo Res. 882/07-R.

2 Dep. Statistics and Operations Research, Faculty of Sciences, University of Alicante, 03071 Alicante, Spain. mgoberna@ua.es

3 UDLA, Cholula, Puebla, Mexico. On leave IMI-BAS, Sofia, Bulgaria. maxim.todorov@udlap.mx

4 Faculty of Economics and I.C.B., National University of Cuyo, Mendoza, 5500, Argentina.vvera@uncu.edu.ar 
we will say "(strong) uniqueness" to mean "uniqueness of the (strong) unique optimal solution" of an LSIO problem.

The problem (1) will also be denoted by $\pi=(a, b, c)$; and by $\Pi=\left(\mathbb{R}^{n} \times \mathbb{R}\right)^{T} \times$ $\mathbb{R}^{n}$ we will denote the linear space of all these kind of problems (the $n$ decision variables and the index set $T$ are fixed), i.e., the result of arbitrary perturbations of the nominal problem $\pi$ preserving $n$ and $T$. In the next section we shall introduce a topology on $\Pi$, which will be called space of parameters.

The pioneering work on uniqueness in linear programming (LP), due to Dantzig (1963, [5]), was completed by Mangasarian (1979, [18]), who provided characterizations not involving optimal basis. Uniqueness and strong uniqueness are no longer equivalent in LSIO, where strong uniqueness plays a crucial role in numerical analysis (together with some regularity conditions it implies superlinear convergence of multiple exchange methods, see [16] - [15]) as well as in sensitivity analysis (it characterizes those problems for which the optimal value is a linear function of the costs on some neighborhood of $c$, as shown in [7]). The literature about uniqueness and strong uniqueness in LSIO up to 1995 was surveyed in [9]. One of the characterizations of the uniqueness in LP included in [18] consists of maintaining the uniqueness under arbitrary but sufficiently small perturbations of the cost vector. The first works on stable (strong) uniqueness are due to Nürnberger ([19] - [20]) and Strauss ([21]); Nürnberger considered the case of continuous LSIO problems and continuous perturbations of the whole triple $(a, b, c)$ ( $\pi$ is a continuous LSIO problem if $T$ is a compact Hausdorff space and the coefficient functions $a$ and $b$ are continuous); while Strauss allowed only perturbations of the RHS function $b$. In this last setting, Cánovas et al. (2007, [3]) have recently shown that Nürnberger condition ([19, Condition (2) in Thm. 1.4]) turns out to be equivalent to the metric regularity of the inverse of the optimal set mapping. In 1998, Helbig and Todorov ([14]) characterized the stable (strong) uniqueness for LSIO problems such that the set of the LHS coefficients of the constraints is bounded by means of a suitable Nürnberger-type condition. Under the same assumption on the boundedness of the LHS coefficients, Goberna, López and Todorov $(2003,[12])$ proved that the set of problems with strongly unique optimal solution contains an open and dense subset of the set of solvable problems. This is a generic result and in view of it we can say that most (in a topological sense) solvable problems satisfying this boundedness condition have a strongly unique optimal solution.

In this paper we analyze the stable (strong) uniqueness of problems which are not upper bounded in the sense of [14], but satisfy a suitable property, e.g., positive distance from the origin to the set of LHS coefficients, or absolutely stability in the sense that the feasible set remains constant under sufficiently small perturbations. In the first case we adapt to our purpose the idea (already used in 1965 by Charnes, Cooper and Kortanek ([4]) in the con- 
text of duality in LSIO) of improving the properties of a given program by means of suitable reformulations: dividing each nontrivial constraint $a_{t}^{\prime} x \geq b_{t}$ by $\left\|\left(\left(a_{t}, b_{t}\right)\right)\right\|$ (called canonical normalization of $\pi$ ), aggregating redundant constraints until the set of coefficients of the constraints is compact (regularization), and eliminating variables in order to get an equivalent problem with full dimensional feasible set (dimension reduction). Here, we use dimension reduction and scaling (not necessarily canonical normalization), not only for the nominal problem $\pi$ (as in [4]) but for the problems in some neighborhood of $\pi$. In doing this we show a very interesting characterization of the lower semicontinuity of the feasible set mapping at $\pi$ : the affine hull of the feasible set remains constant on some neighborhood of $\pi$, that is $\pi$ is affinely stable. Finally, it is important to remark that the strong uniqueness property is a geometric property essentially related to the shape of the feasible set $F$ and the relative position of the gradient vector $c$, while the stability of the strong uniqueness is related to the representation of the set $F$.

In Section 2 we define a topology on $\Pi$ and state some necessary definitions; Section 3 gives the definition of the extended Nürnberger condition, and recalls some known results about it. In Section 4 we characterize the affine stability and apply it to reduce the dimension of $\pi$. These two sections contain Nürnberger-type necessary conditions for stable strong uniqueness based on Theorem 4.1 in [14]. Section 5 gives Nürnberger-type sufficient conditions when $\pi \notin \Pi_{U B}$, by applying the extended Nürnberger condition to suitable reformulations of $\pi$. Finally, Section 6 characterizes the absolutely stable problems and the subclass of stably strongly unique problems; we also provide a generic result about the absolutely stable problems which are uniquely solvable.

\section{Preliminaries}

We represent by $F$ the feasible set and by $F^{*}$ the optimal set of $\pi$. The problem $\pi$ is consistent if $F \neq \emptyset$ and solvable if $F^{*} \neq \emptyset$.

$\widehat{x} \in F$ is a strong Slater element for $\pi$ if there exists $\varepsilon>0$ such that $a_{t}^{\prime} \widehat{x} \geq b_{t}+\varepsilon$ for all $t \in T$. In this case $\pi$ is said to satisfy the strong Slater condition (SSC). In the particular case that $\pi$ is a continuous LSIO problem the strong Slater elements are the Slater points.

$\pi$ has a unique solution if there exists some $\bar{x} \in F$ such that $F^{*}=\{\bar{x}\}$, this optimal solution being strongly unique if there exists $\alpha>0$ such that

$$
c^{\prime} x \geq c^{\prime} \bar{x}+\alpha\|x-\bar{x}\| \quad \text { for all } x \in F,
$$

where $\|\cdot\|$ stands for the Chebyshev norm. (We will always consider $\mathbb{R}^{n}$ equipped 
with the Chebyshev norm).

We say that $\pi$ is LHS-positively lower bounded if inf $\left\{\left\|a_{t}\right\|, t \in T\right\}>0$ (i.e., $\left\{a_{t}, t \in T\right\}$ does not intersect some neighborhood of the null vector $0_{n}$ ). In the same fashion, $\pi$ is LHS-upper bounded if sup $\left\{\left\|a_{t}\right\|, t \in T\right\}<+\infty$ (with an analogous interpretation, just replacing the origin by the point at infinity). Moreover, we say that $\pi$ is absolutely stable if its feasible set is nonempty and remains constant under arbitrary but sufficiently small perturbations of all the data $(a, b, c)$. In Example 1 of [8], it is shown, for any infinite index set $T$, that $\pi$ admits a reformulation in $\Pi$ (with the same objective function, and feasible set assumed to be bounded) which is absolutely stable.

We consider the following subsets of parameters:

$\Pi_{C}=\{\pi \in \Pi: \pi$ is consistent $\}$,

$\Pi_{S S}=\{\pi \in \Pi: \pi$ satisfies the strong Slater condition $\}$

$\Pi_{U}=\{\pi \in \Pi: \pi$ has a unique solution $\}$,

$\Pi_{S U}=\{\pi \in \Pi: \pi$ has a strongly unique solution $\}$,

$\Pi_{P L B}=\{\pi \in \Pi: \pi$ is LHS-positively lower bounded $\}$,

$\Pi_{U B}=\{\pi \in \Pi: \pi$ is LHS-upper bounded $\}$,

and

$\Pi_{A S}=\{\pi \in \Pi: \pi$ is absolutely stable $\}$.

Obviously, $\Pi_{S U} \subset \Pi_{U} \subset \Pi_{C}$ and $\Pi_{S S} \subset \Pi_{C}$. On the other hand, it is wellknown that $\pi \in \Pi_{S S}$ if and only if $\pi$ is stable with respect to consistency ([10, Thm. 6.1]). Thus, $\Pi_{A S} \subset \Pi_{S S}$.

Notice that, if $\pi \in \Pi_{U B} \cap \Pi_{S S}$, then $F$ contains interior points, i.e., $F$ is full dimensional. In fact, if $\rho:=\sup \left\{\left\|a_{t}\right\|, t \in T\right\}$ and $a_{t}^{\prime} \bar{x} \geq b_{t}+\varepsilon$ for all $t \in T$, then the ball centered at $\bar{x}$ with radius $\frac{\varepsilon}{n \rho}$ is contained in $F$ by the Cauchy-Schwartz inequality.

For the next definitions we will make use of the following notation for a given set $X$ :

o If $X$ is a nonempty subset of $\mathbb{R}^{n}$, aff $X, \operatorname{conv} X$, cone $X$ and $\operatorname{dim} X$ denote its affine hull, its convex hull, its convex conical hull containing $0_{n}$, and the dimension of aff $X$, respectively.

o If $X$ is a convex cone in $\mathbb{R}^{n}$, its positive polar is denoted by $X^{0}$ and its lineality space by $\operatorname{lin} X$.

○ If $X$ is convex in $\mathbb{R}^{n}$ and $\bar{x} \in X$, we denote by $D(X ; \bar{x})$ the (convex) cone 
of feasible directions of $X$ at $\bar{x}$.

○ If $X$ is a nonempty subset of either $\mathbb{R}^{n}$ or $\Pi$, int $X, \operatorname{cl} X$ and bd $X$ represent its interior, closure, and boundary, respectively.

The data set of the constraint system of $\pi$ is

$$
D:=\left\{\left(\begin{array}{l}
a_{t} \\
b_{t}
\end{array}\right), t \in T\right\}
$$

whereas the characteristic cone of $\pi$ is

$$
K:=\text { cone }\left\{\left(\begin{array}{l}
a_{t} \\
b_{t}
\end{array}\right), t \in T ;\left(\begin{array}{c}
0_{n} \\
-1
\end{array}\right)\right\} .
$$

Let $a \in \mathbb{R}^{n}$ and $b \in \mathbb{R}$. The nonhomogeneous Farkas lemma establishes that $a^{\prime} x \geq b$ is a consequence of the consistent system $\left\{a_{t}^{\prime} x \geq b_{t}, t \in T\right\}$ if and only if $(a, b) \in \mathrm{cl} K$.

Given $\bar{x} \in F$, the constraint $a_{t}^{\prime} x \geq b_{t}$ is active at $\bar{x}$ if $a_{t}^{\prime} \bar{x}=b_{t}$. We denote by $T(\bar{x})$ the set of active indexes at $\bar{x}, T(\bar{x})=\left\{t \in T: a_{t}^{\prime} \bar{x}=b_{t}\right\}$. The cone of active constraints at $\bar{x}$ is cone $\left\{a_{t}, t \in T(\bar{x})\right\}$. The KKT condition $c \in$ cone $\left\{a_{t}, t \in T(\bar{x})\right\}$ is sufficient for $\bar{x} \in F^{*}$ and it is also necessary if some constraint qualification holds (e.g., $\pi$ is a continuous LSIO problem satisfying the Slater condition). The condition $c \in$ int cone $\left\{a_{t}, t \in T(\bar{x})\right\}$ is sufficient for $\bar{x} \in F$ to be a strongly unique optimal solution of $\pi$, and it is also necessary under some constraint qualification (see [10, Theorems 7.1 and 10.6]). We say that $a^{\prime} x \geq b$ is an implicit active constraint at $\bar{x} \in F$ if $a^{\prime} \bar{x}=b$ and $(a, b) \in \operatorname{cl} D$, in which case $a^{\prime} x \geq b$ for all $x \in F$. Each implicit active constraint $a^{\prime} x \geq b$ is characterized just by the vector $a$; in this fashion the set

$$
A(\bar{x}):=\left\{a \in \mathbb{R}^{n}:\left(\begin{array}{c}
a \\
a^{\prime} \bar{x}
\end{array}\right) \in \operatorname{cl} D\right\}
$$

will be called the set of implicit active constraints at $\bar{x}$. Obviously, $\left\{a_{t}, t \in T(\bar{x})\right\} \subset$ $A(\bar{x})$ and the equality holds if $D$ is closed (e.g., if $\pi$ is continuous). Moreover, if $\pi \in \Pi_{U B}$, then $A(\bar{x})$ is bounded for all $\bar{x} \in F$.

From the topological side, we consider $\Pi$ equipped with the pseudometric of the uniform convergence, i.e., given two parameters $\pi_{1}=\left(a^{1}, b^{1}, c^{1}\right)$ and $\pi_{2}=\left(a^{2}, b^{2}, c^{2}\right)$,

$$
d\left(\pi_{1}, \pi_{2}\right):=\max \left\{\left\|c^{1}-c^{2}\right\|, \sup _{t \in T}\left\|\left(\begin{array}{c}
a_{t}^{1} \\
b_{t}^{1}
\end{array}\right)-\left(\begin{array}{c}
a_{t} \\
b_{t}
\end{array}\right)\right\|\right\}
$$

we use the same symbol to mark a perturbation of the nominal problem $\pi$ and its associated objects. 
It is immediate to prove that $\Pi_{U B}, \Pi_{P L B}$ and $\Pi_{A S}$ are open cones, that $\Pi_{U B}$ is closed and that $\Pi_{P L B}$ is dense in $\Pi$. In fact, given $\varepsilon>0$, replacing in $\pi$ each constraint $a_{t}^{\prime} x \geq b_{t}$ such that $\left\|a_{t}\right\|<\frac{2 \varepsilon}{3}$ by another one $\left(a_{t}^{1}\right)^{\prime} x \geq b_{t}$ such that $\frac{\varepsilon}{6}<\left\|a_{t}^{1}\right\|<\frac{\varepsilon}{3}$, maintaining $b$ and $c$, we get a perturbed problem $\pi_{1} \in \Pi_{P L B}$ such that $d\left(\pi_{1}, \pi\right)<\varepsilon$. The only relationship between these three sets is that $\Pi_{U B} \cap \Pi_{A S}=\emptyset$ (this will be a consequence of Theorem 6.1 ).

To analyze the stability of the LSIO problems which have a strongly unique (or just unique) solution is to determine the topological interiors of $\Pi_{S U}\left(\Pi_{U}\right.$, respectively). Since int $\Pi_{S U} \subset \operatorname{int} \Pi_{U} \subset \Pi_{S S}$ only the elements of $\Pi_{S S}$ are relevant in this paper.

\section{Extended Nürnberger condition and known results}

According to [19], given a continuous LSIO problem $\pi$ satisfying the Slater condition, strong uniqueness holds in a neighborhood of $\pi$ (in the topological subspace of $\Pi$ formed by the continuous problems) if and only if there exists $\bar{x} \in F$ that satisfies:

(a) there exists $\left\{\bar{d}_{1}, \ldots, \bar{d}_{n}\right\} \subset\left\{a_{t}, t \in T(\bar{x})\right\}$ such that $c \in$ cone $\left\{\bar{d}_{1}, \ldots, \bar{d}_{n}\right\}$,

and

(b) for any set $\left\{d_{1}, \ldots, d_{n}\right\} \subset\left\{a_{t}, t \in T(\bar{x})\right\}$ such that $c \in$ cone $\left\{d_{1}, \ldots, d_{n}\right\}$, all the subsets of cardinality $n$ of $\left\{c, d_{1}, \ldots, d_{n}\right\}$ are linearly independent.

Condition (a) means that $\bar{x} \in F^{*}$ (by the optimality theorem and Carathéodory's theorem) and both conditions together imply that $c \neq 0_{n}$. A simple algebraic argument shows that (b) can be replaced in the above Nürnberger condition by the following geometric one:

(b') If $\left\{d_{1}, \ldots, d_{n}\right\} \subset\left\{a_{t}, t \in T(\bar{x})\right\}$ and $c \in$ cone $\left\{d_{1}, \ldots, d_{n}\right\}$, then $c \in$ int cone $\left\{d_{1}, \ldots, d_{n}\right\}$.

Nürnberger theorem can be extended from continuous to general LSIO problems by replacing the set of active constraints $\left\{a_{t}, t \in T(\bar{x})\right\}$ by the enlarged set of implicit active constraints at $\bar{x}, A(\bar{x})$, and strengthening the Slater condition by considering the strong Slater condition (SSC).

Definition 3.1 $\pi \in \Pi$ satisfies the Extended Nürnberger condition at $\bar{x} \in F$ if there exists $\left\{\bar{d}_{1}, \ldots, \bar{d}_{n}\right\} \subset A(\bar{x})$ such that $c \in$ cone $\left\{\bar{d}_{1}, \ldots, \bar{d}_{n}\right\}$ and $c \in$ int cone $\left\{d_{1}, \ldots, d_{n}\right\}$ for any set $\left\{d_{1}, \ldots, d_{n}\right\} \subset A(\bar{x})$ such that $c \in$ cone $\left\{d_{1}, \ldots, d_{n}\right\}$. We say that $\pi \in \Pi$ satisfies the Extended Nürnberger condition (ENC for short) if $\pi$ satisfies this condition at some $\bar{x} \in F$. 
Observe that

$$
c \in \text { cone }\left\{\bar{d}_{1}, \ldots, \bar{d}_{n}\right\} \subset \text { cone } A(\bar{x})
$$

implies that $\bar{x} \in F^{*}$ by Corollary 5.5 in [11]. This means that only the points $\bar{x} \in F^{*}$ (contained in bd $F$ if $c \neq 0_{n}$ ) have to be considered when checking ENC.

The next key result is proved in Theorem 4.1 of [14].

Theorem 3.2 Given $\pi \in \Pi_{U B} \cap \Pi_{S S}$, the following statements are equivalent:

(i) $\pi \in \operatorname{int} \Pi_{S U}$.

(ii) $\pi \in \operatorname{int} \Pi_{U}$.

(iii) $\pi$ satisfies ENC.

Observe that (i)-(iii) fail if $c=0_{n}$ because, in this case, $\pi \in \Pi_{U B} \cap \Pi_{S S}$ entails that $F^{*}=F$ is an infinite set, i.e., $\pi \notin \Pi_{U}$. The next two examples show that the assumption $\pi \in \Pi_{U B}$ in Theorem 3.2 is not superfluous.

Example 3.3 Here we present a problem $\pi \in \Pi_{S S} \backslash \Pi_{U B}$ that does not satisfy ENC even though $\pi \in$ int $\Pi_{S U}$, showing that the implications $(i) \Longrightarrow($ iii $)$ and $($ ii $) \Longrightarrow($ iii $)$ do not hold. Let $n=3, T=\mathbb{Z}$, and consider the problem

$$
\begin{aligned}
\pi: \text { Inf } & x_{2} \\
\text { s.t. } & x_{1}+x_{2}+k x_{3} \geq 0, \quad k \geq 0, \\
& -x_{1}+x_{2}+k x_{3} \geq 0, \quad k<0 .
\end{aligned}
$$

Its feasible set is $F=\left\{x \in \mathbb{R}^{3}: \pm x_{1}+x_{2} \geq 0, x_{3}=0\right\}$, and its optimal set is $F^{*}=\left\{0_{3}\right\}$. We have $\pi \notin \Pi_{U B}$ and $\pi \in \Pi_{S S} \cap \Pi_{S U}$. The data set $D$ is discrete (and so it is closed), thus

$$
A\left(0_{3}\right)=\left\{a \in \mathbb{R}^{n}:\left(\begin{array}{l}
a \\
0
\end{array}\right) \in D\right\}=\left\{a_{t}, t \in T\right\} .
$$

Since $c=(0,1,0) \in$ bd cone $\left\{a_{-1}, a_{0}, a_{1}\right\}$, ENC fails at $0_{3}$. Now, for any $\pi_{1}$ such that the $d\left(\pi_{1}, \pi\right)$ is small enough, $\operatorname{dim} F_{1}=\operatorname{dim} F=2$ (because $\pi, \pi_{1} \in$ $\left.\Pi_{S S}\right)$. The nonhomogeneous Farkas lemma implies that $x_{3} \geq 0$ and $-x_{3} \geq 0$ are consequences of the constraints of $\pi_{1}$. Therefore, aff $F_{1}=\left\{x \in \mathbb{R}^{3}: x_{3}=0\right\}$ (see also Theorem 4.1 below). Then there exists $\mu>0$ such that $d\left(\pi_{1}, \pi\right)<\mu$ implies that $F_{1}$ can be expressed as the set of points of the form $\left(x_{1}, x_{2}, 0\right)$ such that $x_{2} \geq \sup \left\{\alpha_{r}^{1} x_{1}+\beta_{r}^{1}, r \in \mathbb{N} ; \gamma_{r}^{1} x_{1}+\delta_{r}^{1}, r \in \mathbb{N}\right\}$, for some $\alpha_{r}^{1}<-\frac{1}{2}$ and $\gamma_{r}^{1}>\frac{1}{2}$ for all $r \in \mathbb{N}$, so that $\pi_{1} \in \Pi_{S U}$. We conclude that $\pi \in \operatorname{int} \Pi_{S U}$.

Example 3.4 This example shows that both implications $($ iii $) \Longrightarrow(i)$ and $($ iii $) \Longrightarrow($ ii $)$ in the previous theorem are not valid in general when $\pi \in$ 
$\Pi_{S S} \backslash \Pi_{U B}$. Consider the problem in $\mathbb{R}^{2}$,

$$
\pi: \operatorname{Inf} x_{2} \text { s.t. } x_{1}+x_{2} \geq 0,-x_{1}+x_{2} \geq 0 \text {, and } k x_{2} \geq-1, k \in \mathbb{N},
$$

with $F^{*}=\left\{0_{2}\right\}$ and $A\left(0_{2}\right)=\{(1,1),(-1,1)\}$. Then, $\pi \in \Pi_{S S} \backslash \Pi_{U B}$ satisfies ENC and it is not (strongly) unique stable. Indeed, for each $\delta>0$, the problem

$$
\pi_{1}: \operatorname{Inf} x_{2} \text { s.t. } x_{1}+x_{2} \geq-\delta,-x_{1}+x_{2} \geq-\delta \text {, and } k x_{2} \geq-1, k \in \mathbb{N} \text {, }
$$

is such that $d\left(\pi, \pi_{1}\right)=\delta$ and $F_{1}^{*}=\left\{\left(x_{1}, 0\right):\left|x_{1}\right| \leq \delta\right\}$.

The proof of $(i i) \Longrightarrow($ iii $)$ in Theorem 3.2 given in [14] remains valid by replacing the condition $\pi \in \Pi_{U B}$ by the weaker one that the set of implicit active constraints at its optimal solution $\bar{x}, A(\bar{x})$, is bounded. So we can state the following Nürnberger-type necessary condition for stable (strong) uniqueness:

Theorem 3.5 If $\pi \in \operatorname{int} \Pi_{U}$, with $F^{*}=\{\bar{x}\}$, and $A(\bar{x})$ is bounded, then $\pi$ satisfies ENC.

\section{Dimension reduction and Nürnberger-type necessary conditions}

As shown in the next section, the full dimension of the feasible set is a desirable property in order to check the stable strong uniqueness of $\pi$. Here we show that, given an LSIO problem $\pi$ satisfying SSC and such that $0<\operatorname{dim} F<n$, it is possible to reformulate $\pi$ in a lower dimensional space in such a way that the equivalent problem (in a sense to be made precise later) possesses a full dimensional feasible set. In doing that we need a new characterization of the stable consistency of $\pi$.

Theorem $4.1 \pi \in \Pi_{C}$ satisfies the strong Slater condition if and only if there exists $\varepsilon>0$ such that aff $F_{1}=$ aff $F$ for all $\pi_{1} \in \Pi$ with $d\left(\pi_{1}, \pi\right)<\varepsilon$.

Proof. Let $\pi \in \Pi_{C}$. Given $a \in \mathbb{R}^{n}$ and $b \in \mathbb{R}$, by the Farkas Lemma, $a^{\prime} x=b$ for all $x \in F$ if and only if $\pm(a, b) \in \operatorname{cl} K$ if and only if $(a, b) \in \operatorname{lin} \operatorname{cl} K$. Accordingly,

$$
\text { aff } F=\left\{x \in \mathbb{R}^{n}: a^{\prime} x=b \text { for all }(a, b) \in \operatorname{lin} \mathrm{cl} K\right\} .
$$

Now we assume that $\pi \in \Pi_{S S}$. By the argument of (ii) $\Rightarrow$ (vii) in [10, Theorem 6.1], there exists $\varepsilon>0$ such that

$$
\operatorname{lin} \operatorname{cl} K_{1}=\operatorname{lin} \operatorname{cl} K
$$


for all $\pi_{1} \in \Pi$ such that $d\left(\pi_{1}, \pi\right)<\varepsilon$. Combining (2) and (3) we get the conclusion.

Conversely, if aff $F_{1}=$ aff $F$ for all $\pi_{1} \in \Pi$ such that $d\left(\pi_{1}, \pi\right)<\varepsilon$, then $\pi \in \operatorname{int} \Pi_{C}=\Pi_{S S}$.

Remark 4.2 Observe that $\pi \in \Pi_{S S}$ and $\operatorname{dim} F=0$ implies that $\operatorname{dim} F_{1}=0$ for any $\pi_{1}$ in some neighborhood of $\pi$, and hence $\pi \in \operatorname{int} \Pi_{S U}$.

A reduced problem. Assume that $\pi$ satisfies SSC and that $\operatorname{dim} F=d$, $1 \leq d \leq n-1$. Let $\varepsilon>0$ be such that aff $F_{1}=$ aff $F$ if $d\left(\pi_{1}, \pi\right)<\varepsilon$. Since the translation of the feasible sets preserves all the properties of the corresponding problems, we can assume without loss of generality that $V:=$ aff $F \subset \mathbb{R}^{n}$ is a linear subspace of dimension $d$. Let $\tilde{\pi}_{1} \in \Pi_{C}$ be the problem obtained by replacing in $\pi_{1}$ the cost vector $c^{1}$ by its orthogonal projection on $V$, say $\widetilde{c}^{1}$. Obviously, $\widetilde{F}_{1}^{*}=F_{1}^{*}$. Without loss of generality we can assume that $x_{d+1}, \ldots, x_{n}$ are linear combinations of $x_{1}, \ldots, x_{d}$ for all $x=\left(x_{1}, \ldots, x_{n}\right) \in V$. Let $\left(x_{d+1}, \ldots, x_{n}\right)^{\prime}=R\left(x_{1}, \ldots, x_{d}\right)^{\prime}$, where $R$ is a $(n-d) \times d$ matrix. The equation $x=M\left(x_{1}, \ldots, x_{d}\right)^{\prime}$, with $M=\left[\begin{array}{c}I_{d} \\ R\end{array}\right]$, where $I_{d}$ represents the $d \times d$ unit matrix, provides the full dimensional problem equivalent to $\pi_{1}$, say $\theta_{1}$, called reduced problem of $\pi_{1}$ :

$$
\theta_{1}: \operatorname{Inf}\left(e^{1}\right)^{\prime}\left(x_{1}, \ldots, x_{d}\right) \text { s.t. }\left(d_{t}^{1}\right)^{\prime}\left(x_{1}, \ldots, x_{d}\right) \geq b_{t}, t \in T
$$

where $e^{1}=M^{\prime} \widetilde{c}^{1}$ and $d_{t}^{1}=M^{\prime} a_{t}^{1}$ for all $t \in T$. We denote by $\Theta$ the parameter space corresponding to index set $T$ and $d$ variables.

It is easy to prove that $\pi \in \Pi_{S U}\left(\pi \in \Pi_{U}\right)$ if and only if $\theta \in \Theta_{S U}\left(\theta \in \Theta_{U}\right.$, respectively) and that $\widehat{x}$ is a strong Slater element for $\pi$ if and only if $\left(\widehat{x}_{1}, \ldots, \widehat{x}_{d}\right)$ is a strong Slater element for $\theta$. Moreover,

$$
d\left(\theta, \theta_{1}\right) \leq(\sqrt{n}\|M\|+1) d\left(\pi, \pi_{1}\right)
$$

It is also obvious that $\pi \in \Pi_{U B}$ implies that $\theta \in \Theta_{U B}$ because

$$
\sup \left\{\left\|d_{t}\right\|, t \in T\right\} \leq \sqrt{n}\|M\| \sup \left\{\left\|a_{t}\right\|, t \in T\right\} \text {. }
$$

However, the LHS-positive lower boundedness of $\pi$ is not inherited by $\theta$, e.g., for $n=2$, if the constraint system is

$$
\frac{1}{k} x_{1}+k x_{2} \geq-1, \quad \frac{1}{k} x_{1}-k x_{2} \geq-1, \quad k \in \mathbb{N},
$$

for which $F=\left\{\left(x_{1}, 0\right): x_{1} \geq-1\right\}$, then $\pi \in \Pi_{P L B} \cap \Pi_{S S}$ but its reduced problem $\theta \notin \Theta_{P L B}$. Observe also that $\pi \notin \Pi_{U B}$ whereas $\theta \in \Theta_{U B}$. 
Proposition 4.3 Let $\pi \in \Pi_{S S}$ be such that $1 \leq \operatorname{dim} F \leq n-1$ and let $\theta \in \Theta$ be its reduced problem. Then, $\pi \in \operatorname{int} \Pi_{S U}$ if and only if $\theta \in \operatorname{int} \Theta_{S U}$.

Proof. Let $\eta>0$ be such that aff $F_{1}=$ aff $F$ for all $\pi_{1} \in \Pi$ with $d\left(\pi_{1}, \pi\right)<\eta$. Suppose that $\varepsilon$ is such that $0<\varepsilon<\eta$ and $\pi_{1} \in \Pi_{S U}$ for any $\pi_{1} \in \Pi$ with $d\left(\pi_{1}, \pi\right)<\varepsilon$. For any $\theta_{1} \in \Theta$ such that $d\left(\theta_{1}, \theta\right)<\frac{\varepsilon}{2}$ there exists $\pi_{1} \in \Pi$ such that $\theta_{1}$ is the reduced problem of $\pi_{1}$ and $d\left(\pi_{1}, \pi\right)<\varepsilon$. Since $\pi_{1} \in \Pi_{S U}, \theta_{1} \in$ $\Theta_{S U}$. Now assume that $\theta \in \operatorname{int} \Theta_{S U}$ and let $0<\varepsilon<\eta$ be such that $\theta_{1} \in \Theta_{S U}$ if $d\left(\theta_{1}, \theta\right)<\varepsilon$. According to (4), if $\pi_{1} \in \Pi$ satisfies $d\left(\pi_{1}, \pi\right)<\frac{\varepsilon}{\sqrt{n}\|M\|+1}$, then $d\left(\theta_{1}, \theta\right)<\varepsilon$, and this yields $\pi_{1} \in \Pi_{S U}$.

Let us revisit Example 3.3, where $\operatorname{dim} F<n=3$. Here aff $F=\left\{x \in \mathbb{R}^{3}: x_{3}=0\right\}$ and the full dimensional equivalent problem is

$$
\theta: \operatorname{Inf} x_{2} \text { s.t. } \quad-x_{1}+x_{2} \geq 0, t \in T_{1} \text { and } x_{1}+x_{2} \geq 0, t \in T_{2} \text {, }
$$

where $T_{1}=\mathbb{Z}_{-} \cup\left\{t_{2}\right\}$ and $T_{2}=\mathbb{Z}_{+} \cup\left\{t_{1}\right\}$. Since $\theta \in \Theta_{U B} \cap \Theta_{S S}$ and satisfies ENC at $0_{2}$, we conclude that $\pi \in \operatorname{int} \Pi_{S U}$.

Proposition 4.4 Let $\pi \in \Pi_{S S}$ be such that $1 \leq \operatorname{dim} F \leq n-1$ and let $\theta \in \Theta$ be its reduced problem. If $\pi \in \operatorname{int} \Pi_{S U}$ and the set of implicit active constraints at the optimal solution of $\theta$ is bounded, then $\theta$ satisfies ENC.

Proof. It is an immediate consequence of Theorem 3.2, applied to $\theta$, and of Proposition 4.3.

\section{Upper bounding scaling and Nürnberger-type sufficient condi- tions}

In order to get sufficient conditions for $\pi \in \operatorname{int} \Pi_{S U}$ when $\pi \notin \Pi_{U B}$ we appeal to suitable scalings of the constraint systems. We say that a mapping $\varphi: \Pi \rightarrow$ $\Pi_{U B}$ is an upper bounding (UB in short) scaling when $\varphi\left(\pi_{1}\right)$ has the same objective function and the same feasible set as $\pi_{1}$, so that the optimal set of $\varphi\left(\pi_{1}\right)$ also coincides with $F_{1}^{*}$ for all $\pi_{1} \in \Pi$. Thus $\varphi(\pi)$ belongs to $\Pi_{S U}$ $\left(\Pi_{U}, \Pi_{C}\right)$ if and only if $\pi$ belongs to $\Pi_{S U}\left(\Pi_{U}, \Pi_{C}\right.$, respectively). Examples of UB-scaling mappings are the canonical normalization (divide each nontrivial constraint $a_{t}^{\prime} x \geq b_{t}$ by $\left.\left\|\left(\left(a_{t}, b_{t}\right)\right)\right\|\right)$ referred to in Section 1 , and the mappings $\nu$ and $\omega$ which associate with $\pi_{1}=\left(a^{1}, b^{1}, c^{1}\right) \in \Pi$ the problems

$$
\nu\left(\pi_{1}\right)=\pi_{1}^{\nu}: \operatorname{Inf} c_{1}^{\prime} x \text { s.t. }\left(a_{t}^{1 \nu}\right)^{\prime} x \geq b_{t}^{1 \nu}, t \in T
$$


with

$$
\left(a_{t}^{1 \nu}, b_{t}^{1 \nu}\right)= \begin{cases}\left(\frac{a_{t}^{1}}{\left\|a_{t}^{1}\right\|}, \frac{b_{t}^{1}}{\left\|a_{t}^{1}\right\|}\right), & \text { if } a_{t}^{1} \neq 0_{n} \\ \left(a_{t}^{1}, b_{t}^{1}\right), & \text { otherwise }\end{cases}
$$

and

$$
\omega\left(\pi_{1}\right)=\pi_{1}^{\omega}: \operatorname{Inf} c_{1}^{\prime} x \text { s.t. }\left(a_{t}^{1 \omega}\right)^{\prime} x \geq b_{t}^{1 \omega}, t \in T,
$$

with

$$
\left(a_{t}^{1 \omega}, b_{t}^{1 \omega}\right)= \begin{cases}\left(\frac{a_{t}^{1}}{\left\|a_{t}^{1}\right\|}, \frac{b_{t}^{1}}{\left\|a_{t}^{1}\right\|}\right), & \text { if }\left\|a_{t}^{1}\right\|>1 \\ \left(a_{t}^{1}, b_{t}^{1}\right), & \text { otherwise }\end{cases}
$$

respectively (we use the same symbol to mark the image of a problem $\pi_{1}$ by a given UB-scaling mapping and its associated objects). These two UB-scalings are useful due to the properties shown in the next result.

Proposition 5.1 (i) $\nu$ is continuous at any $\pi \in \Pi_{P L B}$. Moreover, $\pi^{\nu} \in \Pi_{S S}$ if $\operatorname{dim} F=n$ and no $a_{t}$ is null.

(ii) $\omega$ is continuous. Moreover, $\pi^{\omega} \in \Pi_{S S}$ if $\pi \in \Pi_{S S}$ and $\operatorname{dim} F=n$.

Proof. (i) Let $\pi \in \Pi_{P L B}$ and $\rho>0$ be such that $\left\|a_{t}\right\|>\rho$ for all $t \in T$. Consider any sequence $\left\{\pi_{r}\right\} \subset \Pi$ such that $\pi_{r} \rightarrow \pi \in \Pi_{P L B}$. Given $0<\varepsilon<$ $\rho / 2$, there exists $r_{0} \in \mathbb{N}$ such that $d\left(\pi_{r}, \pi\right)<\varepsilon$ for all $r \geq r_{0}$. Then, for each $t \in T$ and $r \geq r_{0}$, we have $a_{t}^{r} \neq 0_{n}$ and

$$
\begin{aligned}
\left\|\frac{a_{t}}{\left\|a_{t}\right\|}-\frac{a_{t}^{r}}{\left\|a_{t}^{r}\right\|}\right\| & =\frac{\|\| a_{t}^{r}\left\|a_{t}-\right\| a_{t}^{r}\left\|a_{t}^{r}+\right\| a_{t}^{r}\left\|a_{t}^{r}-\right\| a_{t}\left\|a_{t}^{r}\right\|}{\left\|a_{t}\right\|\left\|a_{t}^{r}\right\|} \\
& \leq \frac{\left\|a_{t}^{r}\right\|\left\|a_{t}-a_{t}^{r}\right\|+\|\| a_{t}^{r}\|-\| a_{t}\|\|\left\|a_{t}^{r}\right\|}{\left\|a_{t}\right\|\left\|a_{t}^{r}\right\|} \\
& \leq \frac{\left\|a_{t}-a_{t}^{r}\right\|+\left\|a_{t}^{r}\right\|-\left\|a_{t}\right\|}{\left\|a_{t}\right\|}<\frac{2 \varepsilon}{\rho},
\end{aligned}
$$

so that

$$
\left\|\frac{a_{t}}{\left\|a_{t}\right\|}-\frac{a_{t}^{r}}{\left\|a_{t}^{r}\right\|}\right\|<\frac{2 \varepsilon}{\rho} .
$$

Analogously,

$$
\left|\frac{b_{t}}{\left\|a_{t}\right\|}-\frac{b_{t}^{r}}{\left\|a_{t}^{r}\right\|}\right|<\frac{2 \varepsilon}{\rho} .
$$

From (5) and (6) we conclude that $d\left(\pi_{r}^{\nu}, \pi^{\nu}\right) \leq \frac{2 \varepsilon}{\rho}$ whenever $r \geq r_{0}$. Hence, $\nu$ is continuous at $\pi$. 
Now, assume that $\pi \in \Pi, \operatorname{dim} F=n$, and $a_{t} \neq 0_{n}$ for all $t \in T$. The feasible set of $\pi^{\nu}$ is $F_{\nu}=F$. Since $\pi^{\nu} \in \Pi_{P L B} \cap \Pi_{U B}, \operatorname{dim} F_{\nu}=n$ if and only if $\pi^{\nu}$ satisfies SSC ([11, Proposition 2.1]). Thus $\pi^{\nu}$ satisfies SSC.

(ii) Next we prove that $\omega$ is continuous at an arbitrary $\pi \in \Pi$.

Consider $\left\{\pi_{r}\right\} \subset \Pi$ such that $\pi_{r} \rightarrow \pi$. Let $\varepsilon>0$ and take $r_{0} \in \mathbb{N}$ such that $d\left(\pi_{r}, \pi\right)<\varepsilon$ for all $r \geq r_{0}$. Let $t \in T$ and take $r \geq r_{0}$. Four cases can arise:

(a) $\left\|a_{t}\right\|,\left\|a_{t}^{r}\right\| \leq 1$. Since $\left(a_{t}^{r \omega}, b_{t}^{r \omega}\right)=\left(a_{t}^{r}, b_{t}^{r}\right)$ and $\left(a_{t}^{\omega}, b_{t}^{\omega}\right)=\left(a_{t}, b_{t}\right)$, we have

$$
\left\|\left(\begin{array}{c}
a_{t}^{r \omega} \\
b_{t}^{r \omega}
\end{array}\right)-\left(\begin{array}{c}
a_{t}^{\omega} \\
b_{t}^{\omega}
\end{array}\right)\right\| \leq d\left(\pi_{r}, \pi\right)<\varepsilon .
$$

(b) $\left\|a_{t}\right\|,\left\|a_{t}^{r}\right\|>1$. Then $\left(a_{t}^{r \omega}, b_{t}^{r \omega}\right)=\left(a_{t}^{r \nu}, b_{t}^{r \nu}\right)$ and $\left(a_{t}^{\omega}, b_{t}^{\omega}\right)=\left(a_{t}^{\nu}, b_{t}^{\nu}\right)$, and we get the aimed conclusion from (5) and (6).

(c) $\left\|a_{t}\right\| \leq 1<\left\|a_{t}^{r}\right\|$. On one hand,

$$
\left\|a_{t}^{r}\right\|=\left\|a_{t}+a_{t}^{r}-a_{t}\right\| \leq\left\|a_{t}\right\|+\left\|a_{t}^{r}-a_{t}\right\|<1+\varepsilon .
$$

Thus, $0 \leq\left\|a_{t}^{r}\right\|-1<\varepsilon$, which gives

$$
\begin{aligned}
\left\|\frac{a_{t}^{r}}{\left\|a_{t}^{r}\right\|}-a_{t}\right\| & =\frac{\left\|a_{t}^{r}-\right\| a_{t}^{r}\left\|a_{t}\right\|}{\left\|a_{t}^{r}\right\|} \leq\left\|a_{t}^{r}-\right\| a_{t}^{r}\left\|a_{t}\right\| \\
& =\left\|a_{t}^{r}-a_{t}+\left(1-\left\|a_{t}^{r}\right\|\right) a_{t}\right\| \\
& \leq\left\|a_{t}^{r}-a_{t}\right\|+\left(\left\|a_{t}^{r}\right\|-1\right)\left\|a_{t}\right\| \\
& <2 \varepsilon .
\end{aligned}
$$

An analogous argument shows that $\left|\frac{b_{t}^{r}}{\left\|b_{t}^{r}\right\|}-b_{t}\right|<2 \varepsilon$. Hence $d\left(\pi_{r}^{\omega}, \pi^{\omega}\right) \leq 2 \varepsilon$.

(d) $\left\|a_{t}^{r}\right\| \leq 1<\left\|a_{t}\right\|$. The proof is similar to the previous one.

Finally, assume that $\pi \in \Pi_{S S}$ and $\operatorname{dim} F=n$. Then $\pi^{\nu} \in \Pi_{S S}$ by (ii). Let $\widehat{x}, \bar{x} \in \mathbb{R}^{n}, \alpha>0$, and $\beta>0$ be such that $a_{t}^{\prime} \widehat{x} \geq b_{t}+\alpha$ and $\left(a_{t}^{\nu}\right)^{\prime} \bar{x} \geq b_{t}^{\nu}+\beta$ for all $t \in T$. Two cases are possible for any $t \in T$ :

If $\left\|a_{t}\right\| \leq 1,\left(a_{t}^{\omega}, b_{t}^{\omega}\right)=\left(a_{t}, b_{t}\right)$, so that $\left(a_{t}^{\omega}\right)^{\prime} \widehat{x} \geq b_{t}^{\omega}+\alpha$.

If $\left\|a_{t}\right\|>1,\left(a_{t}^{\omega}, b_{t}^{\omega}\right)=\left(a_{t}^{\nu}, b_{t}^{\nu}\right)$, so that $\left(a_{t}^{\omega}\right)^{\prime} \bar{x} \geq b_{t}^{\omega}+\beta$.

Thus, $\left(a_{t}^{\omega}\right)^{\prime}\left(\frac{\widehat{x}+\bar{x}}{2}\right) \geq b_{t}^{\omega}+\frac{1}{2} \min \{\alpha, \beta\}$. 
We conclude that $\pi^{\omega} \in \Pi_{S S}$.

Observe that for any $\bar{\pi} \in \Pi_{U B} \cap \Pi_{P L B}$ with bounded $\left\{b_{t}\right\}_{t \in T}$, the sequence $\pi_{r}:=r^{-1} \bar{\pi}, r=1,2, .$. , converges to the null parameter whereas $\pi_{r}^{\nu}=\bar{\pi}^{\nu}$ for all $r \in \mathbb{N}$. Thus $\nu$ is not continuous on the whole space $\Pi$. On the other hand, $\pi \in \Pi_{S S}$ (the general assumption in any test of stable strong uniqueness) does not guarantee that $\pi^{\nu}, \pi^{\omega} \in \Pi_{S S}$.

Example 5.2 Consider an LSIO problem $\pi$ of a single variable with constraint system $\{k x \geq-1, k \in \mathbb{N} ;-k x \geq-1, k \in \mathbb{N}\}$, whose solution set $i s\{0\}$. Obviously, $\pi \in \Pi_{S S}$. Now, the constraint system of $\pi^{\nu}$, and of $\pi^{\omega}$, is given by $\left\{x \geq \frac{1}{k}, k \in \mathbb{N} ;-x \geq-\frac{1}{k}, k \in \mathbb{N}\right\}$, whose unique solution, 0 , is not a strong Slater element. Thus $\pi^{\nu}, \pi^{\omega} \notin \Pi_{S S}$.

Theorem 5.3 Let $\pi \in \Pi_{S S}$ and let $\varphi$ be a UB-scaling mapping continuous at $\pi$ such that $\varphi(\pi) \in \Pi_{S S}$. If $\varphi(\pi)$ satisfies $E N C$, then $\pi \in \operatorname{int} \Pi_{S U}$.

Proof. Since $\varphi(\pi) \in \Pi_{U B} \cap \Pi_{S S}$ and $\varphi(\pi)$ satisfies ENC, then $\varphi(\pi) \in \operatorname{int} \Pi_{S U}$.

Assume now that $\pi \notin \operatorname{int} \Pi_{S U}$. Let $\left\{\pi_{r}\right\} \subset \Pi \backslash \Pi_{S U}$ be such that $\pi_{r} \rightarrow \pi$. Since $\pi \in \Pi_{P L B} \cap \Pi_{S S}$ and this set is open, $\pi_{r} \in \Pi_{P L B} \cap \Pi_{S S}$ for $r$ large enough. By the continuity assumption, $\varphi\left(\pi_{r}\right) \rightarrow \varphi(\pi) \in$ int $\Pi_{S U}$. This implies that $\varphi\left(\pi_{r}\right) \in \Pi_{S U}$ for $r$ large enough, i.e., $\pi_{r} \in \Pi_{S U}$ for $r$ large enough, in contradiction with $\left\{\pi_{r}\right\} \subset \Pi \backslash \Pi_{S U}$.

Example 5.4 Example 3.4 shows that the EN property is not inherited by $\varphi(\pi)$, even in the case that $\varphi$ is continuous at $\pi$ and the feasible set has full dimension. In fact,

$$
\pi^{\nu}: \operatorname{Inf} x_{2} \text { s.t. } \frac{x_{1}+x_{2}}{\sqrt{2}} \geq 0, \frac{-x_{1}+x_{2}}{\sqrt{2}} \geq 0, \text { and } x_{2} \geq-1 / k, k \in \mathbb{N},
$$

is in $\Pi_{U B} \cap \Pi_{S S}$ and does not satisfy ENC.

The next example shows that the converse statement of Theorem 5.3 is not true, at least for $\varphi=\nu$ and $\varphi=\omega$.

Example 5.5 Consider the problem

$$
\begin{array}{ll}
\pi: \text { Inf } & x_{1}+x_{2} \\
\text { s.t. } & k x_{1} \geq-1, k \in \mathbb{N} \\
& k x_{2} \geq-1, k \in \mathbb{N} \\
& k x_{1}+k x_{2} \geq-1, k \in \mathbb{N} \\
& -k x_{1}-k x_{2} \geq-k-1, k \in \mathbb{N} .
\end{array}
$$


We have $F=\operatorname{conv}\{(0,0),(1,0),(0,1)\}, F^{*}=\left\{0_{2}\right\}, \pi \in \Pi_{P L B} \cap \Pi_{S S}$, and $\pi \in \operatorname{int} \Pi_{S U}$ (the last statement will be justified after Theorem 6.2). Nevertheless, the sets of implicit active constraints at $0_{2}$ relative to $\pi$ and $\pi^{\nu}=\pi^{\omega}$ are $\emptyset$ and $\{(1,1),(1,0),(0,1)\}$, respectively. Thus neither $\pi$ nor $\pi^{\nu}=\pi^{\omega}$ satisfy ENC.

Corollary 5.6 Let $\pi \in \Pi_{P L B} \cap \Pi_{S S}$ be such that $\operatorname{dim} F=n$. If $\pi^{\nu}$ satisfies $E N C$, then $\pi \in \operatorname{int} \Pi_{S U}$.

Corollary 5.7 Let $\pi \in \Pi_{S S}$ be such that $\operatorname{dim} F=n$. If $\pi^{\omega}$ satisfies ENC, then $\pi \in \operatorname{int} \Pi_{S U}$.

Corollary 5.8 Let $\pi \in \Pi_{S S}$ be such that $1 \leq \operatorname{dim} F \leq n-1$ and let $\theta \in \Theta$ be its reduced problem. If $\varphi$ is a UB-scaling mapping continuous at $\theta$ such that $\varphi(\theta) \in \Theta_{S S}$ and $\varphi(\theta)$ satisfies $E N C$, then $\pi \in \operatorname{int} \Pi_{S U}$.

\section{Absolute stability}

In this section we need to consider the feasible set mapping $\mathcal{F}: \Pi \rightrightarrows \mathbb{R}^{n}$, which is the multivalued function that associates with each $\pi_{1} \in \Pi$ its feasible set $F_{1}$. The following concepts are due to Bouligand and Kuratowski (see [1, Section 1.4], or [2]).

$\mathcal{F}$ is lower semicontinuous at $\pi \in \Pi$ (lsc, in brief) if, for each open set $W \subset \mathbb{R}^{n}$ such that $W \cap \mathcal{F}(\pi) \neq \emptyset$, there exists an open set $V \subset \Pi$, containing $\pi$, such that $W \cap \mathcal{F}\left(\pi_{1}\right) \neq \emptyset$ for each $\pi_{1} \in V$. Obviously, $\mathcal{F}$ is lsc at $\pi \notin \operatorname{dom} \mathcal{F}$ and $\pi \in \operatorname{int} \operatorname{dom} \mathcal{F}$ if $\mathcal{F}$ is lsc at $\pi \in \operatorname{dom} \mathcal{F}$.

It is well-known that the feasible set mapping $\mathcal{F}$ is lower semicontinuous at $\pi$ if and only if $\pi \in \Pi_{S S}$ ([10, Thm. 6.1]), which is also equivalent to $\pi$ being stable with respect to consistency.

$\mathcal{F}$ is upper semicontinuous at $\pi \in \Pi$ (usc, in brief) if, for each open set $W \subset \mathbb{R}^{n}$ such that $\mathcal{F}(\pi) \subset W$, there exists an open set $V \subset \Pi$, containing $\pi$, such that $\mathcal{F}\left(\pi_{1}\right) \subset W$ for each $\pi_{1} \in V$. If $\mathcal{F}$ is usc at $\pi \notin \operatorname{dom} \mathcal{F}$, then $\pi \in \operatorname{int}(\Pi \backslash \operatorname{dom} \mathcal{F})$.

$\mathcal{F}$ is closed at $\pi \in \operatorname{dom} \mathcal{F}$ if for all sequences $\left\{\pi_{r}\right\} \subset \Pi$ and $\left\{z_{r}\right\} \subset \mathbb{R}^{n}$ satisfying $z_{r} \in \mathcal{F}\left(\pi_{r}\right)$ for all $r \in \mathbb{N}, \pi_{r} \rightarrow \pi$, and $z_{r} \rightarrow z$, one has $z \in \mathcal{F}(\pi)$. If $\mathcal{F}$ is usc at $\pi \in \operatorname{dom} \mathcal{F}$ and $\mathcal{F}(\pi)$ is closed, then $\mathcal{F}$ is closed at $\pi$. Conversely, if $\mathcal{F}$ is closed and locally bounded at $\pi \in \operatorname{dom} \mathcal{F}$ (i.e., if there exists a neighborhood of $\pi$, say $V$, and a bounded set in $\mathbb{R}^{n}$ containing $\mathcal{F}\left(\pi_{1}\right)$ for every $\left.\pi_{1} \in V\right)$, then $\mathcal{F}$ is usc at $\pi$. 
$\mathcal{F}$ is lsc (usc, closed) if it is lsc (usc, closed) at $\pi$ for all $\pi \in \Pi$.

Finally, recall that $\pi$ is absolutely stable (in the feasible set sense) if there exists $\delta>0$ such that $\mathcal{F}\left(\pi_{1}\right)=F$ for all $\pi_{1} \in \Pi$ with $d\left(\pi_{1}, \pi\right)<\delta$. This is the strongest conceivable form of stability of the feasible set mapping $\mathcal{F}: \Pi \rightrightarrows \mathbb{R}^{n}$.

The following result gives necessary and sufficient conditions for $\pi$ being $a b$ solutely stable when the feasible set $F$ is bounded.

Theorem 6.1 Let $\pi \in \Pi_{C}$. If $\pi$ is absolutely stable then there exists a positive scalar $\varepsilon$ such that:

(i) $a_{t}^{\prime} x \geq b_{t}+\varepsilon$ for all $t \in T$ and for all $x \in F$, and

(ii) for each $x \notin F$ there exists some $t \in T$ such that $a_{t}^{\prime} x<b_{t}-\varepsilon$.

Conversely, (i) and (ii) are sufficient conditions for $\pi$ being absolutely stable if $F$ is bounded.

Moreover, $\pi$ absolutely stable implies that $\pi \in \Pi_{S S} \backslash \Pi_{U B}$, and that $F$ is bounded if, in addition, $\pi \notin \Pi_{P L B}$ and $a_{t} \neq 0_{n}$ for all $t \in T$.

Proof. Assume that $\pi$ is absolutely stable and let $\delta>0$ be such that $F_{1}=F$ if $d\left(\pi_{1}, \pi\right)<\delta$. Put $\varepsilon=\delta / 2$ and consider the problems $\pi_{1}$ and $\pi_{2}$ with $a_{t}^{1}=a_{t}, b_{t}^{1}=b_{t}+\varepsilon$, and $a_{t}^{2}=a_{t}, b_{t}^{2}=b_{t}-\varepsilon$, for $t \in T$, respectively. Then $F_{1}=F_{2}=F \neq \emptyset$. Hence, by taking any $x \in F=F_{1},(i)$ is satisfied. Moreover, if $x \notin F$ then $x \notin F_{2}$, which yields (ii).

Suppose now that $F$ is bounded and that there exists $\varepsilon>0$ satisfying (i) and (ii). $\mathcal{F}$ is upper semicontinuous at $\pi$ by the boundedness of $F$ ([10, Corollary 6.2.1]). Let $\rho, \gamma>0$ be such that $F_{1}$ is contained in the open ball centered at $0_{n}$ with radius $\rho, B\left(0_{n} ; \rho\right)$, if $d\left(\pi_{1}, \pi\right)<\gamma$.

Put

$$
\delta=\frac{1}{2} \min \left\{\varepsilon(n+1)^{-1 / 2}(\rho+1)^{-1}, \gamma\right\}>0 .
$$

If $x \in B\left(0_{n} ; \rho\right)$ and $\pi_{1}$ satisfies $d\left(\pi_{1}, \pi\right)<\delta$, we have

$$
\left\|\left(\begin{array}{c}
x \\
-1
\end{array}\right)\right\| \leq \rho+1
$$

and so, by the Cauchy-Schwartz inequality,

$$
\left|\left[\left(\begin{array}{c}
a_{t}^{1} \\
b_{t}^{1}
\end{array}\right)-\left(\begin{array}{c}
a_{t} \\
b_{t}
\end{array}\right)\right]^{\prime}\left(\begin{array}{c}
x \\
-1
\end{array}\right)\right| \leq \delta(n+1)^{1 / 2}(\rho+1)<\varepsilon .
$$

If $x \in F$, given any $t \in T,\left(\begin{array}{c}a_{t} \\ b_{t}\end{array}\right)^{\prime}\left(\begin{array}{c}x \\ -1\end{array}\right) \geq \varepsilon$ by assumption $(i)$; hence (7) yields $\left(\begin{array}{c}a_{t}^{1} \\ b_{t}^{1}\end{array}\right)^{\prime}\left(\begin{array}{c}x \\ -1\end{array}\right)>0$, so that $x \in F_{1}$. Thus $F \subset F_{1}$.

In the case $x \notin F$, take $t \in T$ such that $a_{t}^{\prime} x<b_{t}-\varepsilon$, i.e., $\left(\begin{array}{c}a_{t} \\ b_{t}\end{array}\right)^{\prime}\left(\begin{array}{c}x \\ -1\end{array}\right)<-\varepsilon$. 
Reasoning as before we get $\left(\begin{array}{c}a_{t}^{1} \\ b_{t}^{1}\end{array}\right)^{\prime}\left(\begin{array}{c}x \\ -1\end{array}\right)<0$, so that $x \notin F_{1}$. Hence $F_{1} \subset F$. Therefore $F_{1}=F$.

Now, if $\pi$ is absolutely stable, then $\pi \in \operatorname{int} \Pi_{C}=\Pi_{S S}$. Moreover, there exists $\delta>0$ such that $F_{1}=F_{2}$ for all $\pi_{1}, \pi_{2} \in \Pi$ with $d\left(\pi_{1}, \pi\right)<\delta$ and $d\left(\pi_{2}, \pi\right)<\delta$. Then, according to Proposition 4.1 in [13], the set $\left\{\left\|a_{t}\right\|, t \in T\right\}$ cannot be bounded. In particular the index set $T$ is infinite.

Suppose, additionally, that $\pi \notin \Pi_{P L B}$ and $a_{t} \neq 0_{n}$ for all $t \in T$. Let $\delta>0$ be such that $F_{1}=F$ if $d\left(\pi_{1}, \pi\right) \leq \delta$. Let $t_{1}, s_{1}, \ldots, t_{n}, s_{n}$ be non repeated elements of $T$ such that $\left\|a_{t_{i}}\right\|<\frac{\delta}{2}$ and $\left\|a_{s_{i}}\right\|<\frac{\delta}{2}, i=1, \ldots, n$. Let $\pi_{1}$ be the problem obtained by replacing $a_{t_{i}}^{\prime} x \geq b_{t_{i}}$ by $\frac{\delta}{2} x_{i} \geq b_{t_{i}}$ and $a_{s_{i}}^{\prime} x \geq b_{s_{i}}$ by $-\frac{\delta}{2} x_{i} \geq b_{s_{i}}$, $i=1, \ldots, n$. Since $d\left(\pi_{1}, \pi\right) \leq \delta$, any $x \in F=F_{1}$ satisfies $\frac{2}{\delta} b_{t_{i}} \leq x_{i} \leq-\frac{2}{\delta} b_{s_{i}}$, $i=1, \ldots, n$, i.e., $F$ is contained in a box.

Observe that condition (i) in Theorem 6.1 can be seen as a uniform strong Slater condition. The problem $\pi$ in $\mathbb{R}$ with the unique constraint $0 x \geq-1$ satisfies conditions (i) and (ii), but it is not absolutely stable; its feasible set $F$ is unbounded. Notice that the feasible set mapping $\mathcal{F}$ is upper semicontinuous at $\pi$ because $n=1$, so the condition " $F$ is bounded" can not be substituted by " $\mathcal{F}$ is upper semicontinuous at $\pi$ ".

The characterization of stable strong uniqueness for absolutely stable problems is quite simple. The next result can be seen as the LSIO counterpart of the Mangasarian characterization of uniqueness in LP in terms of perturbations of the cost vector.

Theorem 6.2 Let $\pi \in \Pi_{A S}$. Then $\pi \in \operatorname{int} \Pi_{S U}$ if and only if $\pi \in \Pi_{S U}$.

Proof. Let $\delta>0$ be such that $F_{1}=F$ if $d\left(\pi_{1}, \pi\right)<\delta$. Assume that $\pi \in \Pi_{S U}$ and let $\bar{x} \in F$ be the strongly unique optimal solution of $\pi$. This happens if and only if $c \in \operatorname{int} D(F ; \bar{x})^{0}([10$, Theorem 10.5]). Thus, there exists $\rho>0$ such that $B(c ; \rho) \subset D(F ; \bar{x})^{0}$. Let $\varepsilon=\min \{\delta, \rho\}$. Then $d\left(\pi_{1}, \pi\right)<\varepsilon$ implies $c^{1} \in \operatorname{int} D(F ; \bar{x})^{0}=\operatorname{int} D\left(F_{1} ; \bar{x}\right)^{0}$, so that $\pi_{1} \in \Pi_{S U}$.

Consider again Example 5.5, where $\pi \in \Pi_{S U}$ and

$$
F=\operatorname{conv}\{(0,0),(1,0),(0,1)\}=\left\{x \in \mathbb{R}^{2}: x_{1} \geq 0, x_{2} \geq 0, x_{1}+x_{2} \leq 1\right\}
$$

By Theorem 6.2, $\pi \in \operatorname{int} \Pi_{S U}$ provided $\pi \in \Pi_{A S}$. Since $(0,0),(1,0)$, and $(0,1)$ satisfy $a_{t}^{\prime} x \geq b_{t}+1$ for all $t \in T$, condition (i) in Theorem 6.1 hods with $\varepsilon=1$. Moreover, if $x \notin F$ at least one of the three constraints in (8) fails. Assume, e.g., that $x_{1}+x_{2}>1$. In this case there exists $r \in \mathbb{N}$ such that $r\left(x_{1}+x_{2}-1\right)>2$, and so condition (ii) also holds with $\varepsilon=1$ (the other two cases, $x_{1}<0$ and $x_{2}<0$, are similar). Thus $\pi \in \Pi_{A S}$, which implies that $\pi \in \operatorname{int} \Pi_{S U}$. 
Now consider that $\pi \in \Pi_{A S}$. Observe that, according to Corollary 5.5 in [11], if there exists $\bar{x} \in F$ such that $c \in$ int cone $A(\bar{x})$, then $\bar{x}$ is a strongly unique solution of $\pi$ and so $\pi \in \operatorname{int} \Pi_{S U}$. On the other hand, if $n=2, c=(0,1)$, and $F=\operatorname{clconv}\left\{\left( \pm \frac{1}{r}, \frac{1}{r^{2}}\right), r \in \mathbb{N}\right\}$, we have $\pi \in \Pi_{U} \backslash\left(\right.$ int $\left.\Pi_{U}\right)$. Thus, it is not possible to replace $\Pi_{S U}$ by $\Pi_{U}$ in Theorem 6.2. Finally we give a generic result.

Theorem 6.3 Let $\pi \in \Pi_{A S}$ and let $W$ be a neighborhood of $\pi$ where $\mathcal{F}$ is constant. Then there exists a dense and $G_{\delta}$ subset of $W$ formed by problems which have at most one optimal solution.

Proof. Suppose that $F$ is the feasible set of $\pi$. For each $c \in \mathbb{R}^{n}$, consider the parametric problem $P(c): \operatorname{Inf} c^{\prime} x$ subject to $x \in F$. The property will follows by showing that there exists a dense and $G_{\delta}$ subset $A$ of $\mathbb{R}^{n}$ such that the optimal set of $P(c), F^{*}(c)$, satisfies $\left|F^{*}(c)\right| \leq 1$ for all $c \in A$. Given $m \in \mathbb{N}$, let $F_{m}:=F \cap \mathrm{cl} B\left(0_{n} ; m\right)$, which is nonempty for $m$ large enough, say $m \geq m_{0}$. Consider the problem $P_{m}(c): \operatorname{Inf} c^{\prime} x$ subject to $x \in F_{m}$ and its optimal set mapping $\mathcal{F}_{m}^{*}: \mathbb{R}^{n} \rightrightarrows \mathbb{R}^{n}$. This mapping $\mathcal{F}_{m}^{*}$ is uniformly bounded and closed, so it is usc and Fort's theorem ([6], [17]) implies that it is also lsc on some dense and $G_{\delta}$ subset $A_{m}$ of $\mathbb{R}^{n}$. The set $A:=\cap_{m \geq m_{0}} A_{m}$ is dense and $G_{\delta}$ because $\mathbb{R}^{n}$ is a Baire space. Now we will prove that $\left|F^{*}(c)\right| \leq 1$ for any $c \in A$. Suppose on the contrary that there exist $c \in A$, and $x^{*}, y^{*} \in F^{*}(c)$, $x^{*} \neq y^{*}$. Taking $m>\max \left\{\left\|x^{*}\right\|,\left\|y^{*}\right\|\right\}$, it follows that $x^{*}, y^{*} \in F_{m}^{*}(c)$.

Consider $\varepsilon>0$ small enough so that $\left(y^{*}-x^{*}\right)^{\prime}\left(z-x^{*}\right)>0$ for all $z \in$ $B\left(y^{*} ; \varepsilon\right)$. Put $c^{k}:=c+\frac{1}{k}\left(y^{*}-x^{*}\right)$ and observe that, for any $z \in F \cap B\left(y^{*} ; \varepsilon\right)$,

$$
\left(c^{k}\right)^{\prime}\left(z-x^{*}\right)=c^{\prime}\left(z-x^{*}\right)+\frac{1}{k}\left(y^{*}-x^{*}\right)^{\prime}\left(z-x^{*}\right)>0 .
$$

Hence $\left(c^{k}\right)^{\prime} z>\left(c^{k}\right)^{\prime} x^{*}$ and so $z \notin \mathcal{F}_{m}^{*}\left(c^{k}\right)$, a contradiction with $\mathcal{F}_{m}^{*}$ being lsc at $c$, because $\mathcal{F}_{m}^{*}(c) \cap B\left(y^{*} ; \varepsilon\right) \neq \emptyset$.

The final example shows that we cannot replace "unique" by "strongly unique" in Theorem 6.3. It also shows that $\left(\operatorname{int} \Pi_{S U}\right) \cap \Pi_{S S} \varsubsetneqq\left(\right.$ int $\left.\Pi_{U}\right) \cap \Pi_{S S}$, so that $\operatorname{int} \Pi_{S U} \varsubsetneqq \operatorname{int} \Pi_{U}$.

Example 6.4 Let $n=2$, and $T=\mathbb{N} \times[0,2 \pi]$. If $c$ is any non null vector in $\mathbb{R}^{2}, a_{(m, \alpha)}=-(m \cos \alpha, m \sin \alpha)$ and $b_{(m, \alpha)}=-m-1$ for all $(m, \alpha) \in T$. Obviously, $\pi \in \Pi_{P L B} \cap \Pi_{S S}$. Since $\left\|a_{(m, \alpha)}\right\|=m, a_{(m, \alpha)}^{\nu}=-(\cos \alpha, \sin \alpha)$ and $b_{(m, \alpha)}^{\nu}=-1-\frac{1}{m}$, we have

$$
\begin{aligned}
F=F_{\nu} & =\bigcap_{m=1}^{\infty}\left\{x \in \mathbb{R}^{2}: x_{1}^{2}+x_{2}^{2} \leq\left(1+\frac{1}{m}\right)^{2}\right\} \\
& =\left\{x \in \mathbb{R}^{2}: x_{1}^{2}+x_{2}^{2} \leq 1\right\}
\end{aligned}
$$


and $\left\{\pi, \pi^{\nu}\right\} \subset \Pi_{U} \backslash \Pi_{S U}$. Since $A^{\nu}(\bar{x})=\{-\bar{x}\}$ for all $\bar{x} \in$ bd $F, \pi^{\nu}$ does not satisfy ENC. Nonetheless we will show that $\pi$ is absolutely stable. In fact, given $x \in F$ and $t=(m, \alpha) \in T$, we have

$$
a_{t}^{\prime} x-b_{t}=1+m\left[1-\left((\cos \alpha) x_{1}+(\sin \alpha) x_{2}\right)\right] \geq 1,
$$

and given $x \notin F$, there exists $\alpha \in[0,2 \pi]$ such that $(\cos \alpha) x_{1}+(\sin \alpha) x_{2}>1$. Then there exists $m \in \mathbb{N}$ such that $m\left[(\cos \alpha) x_{1}+(\sin \alpha) x_{2}-1\right]>2$, i.e.,

$$
(-m \cos \alpha) x_{1}+(-m \sin \alpha) x_{2}<-(m+1)-1 \text {. }
$$

Hence conditions ( $i$ ) and (ii) in Theorem 6.1 hold for $\varepsilon=1$. On the other hand, since $c^{1} \neq 0_{n}$ in some neighborhood of $\pi$ we conclude that $\pi \in \operatorname{int} \Pi_{U}$.

\section{References}

[1] Aubin, J.P., and Frankowska H., Set-Valued Analysis, Birkhäuser, Boston, 1990.

[2] Bank, B., Guddat, J., Klatte, D., Kummer, B., and Tammer, K., Non-Linear Parametric Optimization, Birkhäuser-Verlag, Basel, 1983.

[3] Cánovas, M.J., Klatte, D., López, M.A., and Parra, J., Metric regularity in convex semi-infinite optimization under canonical perturbations, SIAM J. Optim., Vol. 18, pp. 717-732, 2007.

[4] Charnes, A., Cooper, W.W., and Kortanek, K.O., On representations of semiinfinite programs which have no duality gaps, Manag. Sci., Vol. 12, pp. 113-121, 1965 .

[5] Dantzig, G.B., Linear Programming and Extensions, Princeton University Press, Princeton, 1963.

[6] Fort, M.K., A unified theory of semi-continuity, Duke Math. J., Vol. 16, pp. 237-246, 1949.

[7] Goberna, M.A., Gómez, S., Guerra, F., and Todorov, M.I., Sensitivity analysis in linear semi-infinite programming: perturbing cost and right-handside coefficients, European J.Oper. Res., Vol. 181, pp. 1069-1085, 2007.

[8] Goberna, M.A., Larriqueta, M., and Vera de Serio, V.N., On the stability of the boundary of the feasible set in linear optimization, Set-Valued Anal., Vol. 11, pp. 203-223, 2003.

[9] Goberna, M.A., López, M.A., and Todorov, M.I., Unicity in linear optimization, J. Optim. Theory Appl., Vol. 86, pp. 37-56, 1995.

[10] Goberna, M.A., and López, M.A., Linear Semi-Infinite Optimization, J. Wiley, Chichester, 1998. 
[11] Goberna, M.A., López, M.A., and Todorov, M.I., Extended active constraints in linear optimization with applications, SIAM J. Optim., Vol. 14, pp. 608-619, 2003.

[12] Goberna, M.A., López, M.A., and Todorov, M.I., A generic result in linear semi-infinite optimization, Appl. Math. Optim., Vol. 48, pp. 181-193, 2003.

[13] Goberna, M.A., Jeyakumar, V., and Dinh, N., Dual characterizations of set containments with strict inequalities, J. Global Optim., Vol. 34, pp. 33-54, 2006.

[14] Helbig, S., and Todorov, M.I., Unicity results for general linear semi-infinite optimization problems using a new concept of active constraints, Appl. Math. Optim., Vol. 38, pp. 21-43, 1998.

[15] Hettich, R., and Zencke, P., Numerische Methoden der Approximation und semi-infiniten Optimierung, B. G. Teubner, Stuttgart, 1982.

[16] Hettich, R., A review of numerical methods for semi-infinite optimization. Semi-Infinite Programming and Applications, Edited by A.V. Fiacco and K.O. Kortanek, Springer-Verlag, Berlin, pp. 158-178, 1983.

[17] Kenderov, P.S., Continuity-like properties of set-valued mappings, Serdica, Vol. 9, pp. 149-160, 1983.

[18] Mangasarian, O., Uniqueness of solutions in linear programming, Linear Algebra Appl., Vol. 25, pp. 151-162, 1979.

[19] Nürnberger, G., Unicity in semi-infinite optimization. Parametric Optimization and Approximation, Edited by B. Brosowski, and F. Deutsch, Birkhäuser, Basel, pp. 231-247, 1985.

[20] Nürnberger, G., Strong unicity in nonlinear parametric optimization. Parametric Optimization and Related Topics, Edited by J. Guddat, H.Th. Jongen, B. Kummer, and F. Nozicka, Akademie-Verlag, Berlin, pp. 316-326, 1987.

[21] Strauss, H., Uniqueness in linear semi-infinite optimization, J. Approx. Theory, Vol. 75, pp. 198-213, 1993. 